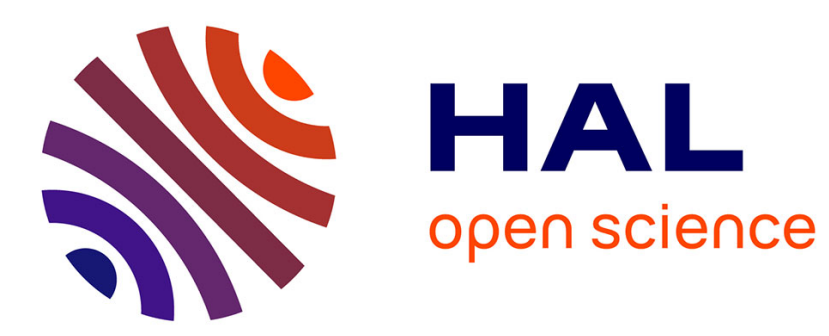

\title{
Simple signaling games of sexual selection (Grafen's revisited)
}

Pierre Bernhard, Frédéric Marie Hamelin

\section{To cite this version:}

Pierre Bernhard, Frédéric Marie Hamelin. Simple signaling games of sexual selection (Grafen's revisited). Journal of Mathematical Biology, 2014, 69 (6-7), pp.1719-1742. 10.1007/s00285-013-0745-8 . hal-00933425

\section{HAL Id: hal-00933425 \\ https://hal.inria.fr/hal-00933425}

Submitted on 3 Dec 2014

HAL is a multi-disciplinary open access archive for the deposit and dissemination of scientific research documents, whether they are published or not. The documents may come from teaching and research institutions in France or abroad, or from public or private research centers.
L'archive ouverte pluridisciplinaire HAL, est destinée au dépôt et à la diffusion de documents scientifiques de niveau recherche, publiés ou non, émanant des établissements d'enseignement et de recherche français ou étrangers, des laboratoires publics ou privés. 


\title{
Simple signaling games of sexual selection (Grafen's revisited)
}

\author{
Pierre Bernhard* and Frédéric M. Hamelin ${ }^{\dagger}$
}

October 19, 2013

\begin{abstract}
We investigate several versions of a simple game of sexual selection, to explore the role of secondary sexual characters (the "handicap paradox") with the tools of signaling theory. Our models admit closed form solutions. They are very much inspired by Grafen (1990)'s seminal companion papers. By merging and simplifying his two approaches, we identify a not so minor artifact in the seminal study. We propose an alternative model to start with Grafen's sexual selection theory, with several similarities with Getty (1998).
\end{abstract}

\section{Introduction}

Sexual selection was introduced by Charles Darwin as a mechanism complementary to natural selection in shaping evolution. In short, the number of offspring to any individual is a function of both of its survivorship, the source of natural selection, and its mating success, the source of sexual selection.

The investigation of sexual selection has been dominated by that of the role of secondary sexual characters, and by the "handicap paradox" — not to be mistaken with the "handicap principle" to be described hereafter. The paradox is that secondary sexual characters that seem to attract individuals of the other sex, often male characters attracting females, seem excessively developed, with a negative impact on the viability of the animals bearing them. Since females attracted by these males run the risk of having ill-adapted male progeny, such a preference should be counter-selected.

\footnotetext{
*Emeritus senior scientist, BIOCORE team, INRIA-Sophia Antipolis-Méditerranée, France.

${ }^{\dagger}$ Lecturer at AGROCAMPUS OUEST, Rennes, France.
} 
There has been a very large literature discussing this topic, proposing several possible mechanisms to explain the paradox. We refer to [11,9] for classifications of the proposed mechanisms, and to the review [12] and the bibliography therein. Over-simplifying, two mechanisms are in competition in the literature: the so called "Fisherian runaway" dynamic mechanism, see $[4,13]$, and Zahavi's static, or equilibrium, "handicap principle" $[18,8,7]$.

Zahavi's intuition was made precise in a mathematical model by Grafen [8, 7]. In [8], he develops a model in terms of population genetics. In [7], the handicap principle results from an analysis of the role of secondary sexual characters as signals, in the framework of signaling theory, a branch of game theory. This model is one of direct benefits in the classification of [12] (or direct selection D in the classification of [11]). Yet, in [12], the handicap principle is quoted as belonging to the family of indirect benefits, more specifically the "good gene" mechanism ${ }^{1}$.

In this study, and consistent with, e.g. [5], we will not need to decide whether the quality of males is genetic or environment dependent. The only thing that matters is that females have a benefit in reproductive efficiency to mate with good males, either because these are more fertile (a "direct" effect) leading to a larger progeny at the first generation, or because their own offspring will have a larger fitness (an indirect "good gene" effect: larger survivorship or better mating success - "sexy son"-), leading to a larger progeny at the second generation.

We shall develop an asymmetrical model where males signal to attract females. It is known that this is not always the case; the reverse exists, and even cases of mutual signaling are well documented, a most famous example being in the Great Crested Grebe Podiceps cristatus, which form stable pairs. But, following the main trend in the literature, we will assume that males keep foraging for mates, and that they have a potential reproduction rate much larger than what the number of females allow, so that one may consider that they can mate an arbitrary number of times. As a consequence, even if females are unequally fertile, there is no point for males to be choosy, and therefore no evolutionary pressure on females to develop signals of their quality. In contrast, females are assumed to mate only once per breeding season. Hence if males are of unequal qualities, there is a reproductive benefit for females in being choosy, and therefore evolutionary pressure on males to develop signals of their quality.

In this line of thought, our model may be viewed as a simple synthesis of Grafen's [7, 8] to be revisited. As in [7], we will use reproductive efficiency as the payoff of the game; however, inspired by the companion paper [8], we will use a mechanistic model, with explicit mating dynamics, for reproductive efficiency.

\footnotetext{
${ }^{1}$ However, the explanation of the handicap principle in [12] contains a mistake concerning the transitivity of positive correlations.
} 
One major simplification compared to Grafen's model [8] is that we do not allow females' fecundity to vary during the breeding season. A minor difference is that we deal with viability in a somewhat different way. While Grafen's example deals with a probability to survive the pre-breading season, which better fits semelparous species, we deal with an expected number of breeding seasons, which extends the model to iteroparous species as well ([15], [5]). We also use from the start two simplifications of Grafen's model which he uses in the example that he solves numerically: a uniform distribution of males' qualities and a fecundity of females proportional to their mate's quality. As a benefit, we gain explicit solutions, through significantly simpler derivations.

We investigate first a model where these are essentially the only differences with Grafen's. Then we propose a second model, using ideas close to those of [5], but again more explicit, to circumvent an undesirable feature in Grafen's model pertaining to the lower bound of admissible males' quality.

In Section 2, we give a quick account of the basics of signaling game theory, including the very simple derivation of what we call the handicap principle. In Section 3, we develop two variants of each of our two models of sexual selection, which all turn out to have explicit equilibrium strategies, simplifying the discussion of the model's conclusions.

\section{Signaling}

\subsection{Definitions}

\subsubsection{Motivation and intuition}

We consider the secondary sexual characters under investigation as "signals", i.e. means by which a male communicates to females his "quality", a "private" parameter that females cannot observe directly, in order to attract them. Hence their primary role is of carrying information.

The investigation of situations where two "players", here males and females, interact, and where one has to rely on the observed actions of the other one to recover some missing information about him, is the object of signaling game theory. We investigate equilibrium situations, which needs not be always the case in biological signaling. We assume that all the decision and signal variables range over a continuum, plus some technical assumptions to be given hereafter. The "private" parameter of player one, denoted $\theta$ in the next section, will be males' quality $q$ in the sexual selection models.

In essence, the explanation of the handicap paradox via signaling theory, the so-called "handicap principle", simply states that, if reproductive success is max- 
imized by the selective pressure of evolution, and if it is a function of both life expectancy and mating success, and if the marginal effect of the signal size on mating success is positive, its marginal effect on survivorship has to be negative to balance the first one and get zero marginal value at equilibrium ([5]).

Another intuition, which is equally valid, is that if the signal were "cheap", a low-quality male could, for free, mimic a high quality one and so doing improve his pay-off, contradicting the equilibrium hypothesis.

In the next subsections, we give a short account of signaling theory, that we need to proceed.

\subsubsection{A signaling game}

A simple signaling game is as follows. There are two players, 1 and 2, choosing their decisions $u_{1}$ and $u_{2}$ in decision sets $U_{1}$ and $U_{2}$ respectively, most often sets of mixed strategies.

The payoffs of the players are functions $J_{i}\left(\theta, u_{1}, u_{2}\right), i=1,2$, of the decisions and of a parameter $\theta$ belonging to some set $\Theta$. But $\theta$ is a "private information" of player 1, i.e. player 1 is able to choose his control via a strategy $\psi_{1}$ as $u_{1}=\psi_{1}(\theta)$, while player 2 does not know $\theta$.

The game proceeds as follows: player 1 plays first. His decision $u_{1}$ creates, possibly via a noisy — probabilistic - mechanism, a signal $s$ observed by player 2. Then player 2 plays, and he is allowed to choose his decision $u_{2}$ as a function of the signal, say $u_{2}=\psi_{2}(s)$.

The rules of the game, the sets $\Theta, U_{1}, U_{2}$, the criteria $J_{1}$ and $J_{2}$, and the mechanism that generates $s$ as a function of $\theta$ and $u_{1}$, leading to a probability law $\mathbb{P}_{1}\left(s \mid \theta, u_{1}\right)$, are common knowledge. (i.e. both players know them and know that the other one knows, ..., ad infinitum.) But player 2 does not know $\theta$, nor the realizations of the random variables involved in generating $s$.

Finally, we are compelled to adopt Harsanyi's device for games of incomplete information: both players share an a priori distribution law, say $\mathbb{P}_{0}$, upon $\theta$.

The interesting feature of that kind of game is that in making his decision, player 1 should take into account the information about his private data that he is leaking to player 2 through his own decision.

Pure signaling game We shall consider a simplified form of signaling game, called pure signaling game, where, on the one hand, $J_{2}$ does not depend directly on $u_{1}$, so that player two is only interested in the informational content of $u_{1}$, which, on the other hand, he exactly observes, with no uncertainty. 


\subsubsection{Bayesian equilibrium}

We now describe what we consider as an equilibrium of this game, called a bayesian equilibrium, the natural extension of the Nash equilibrium to signaling games.

First form Player 2 will form a conjecture as to how player 1 has formed his decision, of the form $u_{1}=\chi(\theta)$. Using that conjecture and the signal $s$, he will update his probability distribution over $\theta$ according to Bayes rule, producing a conditional law $\mathbb{P}_{2}(\theta \mid s)$ :

$$
\mathbb{P}_{2}(\theta \mid s)=\frac{\mathbb{P}_{1}(s \mid \theta, \chi(\theta)) \mathbb{P}_{0}(\theta)}{\int \mathbb{P}_{1}(s \mid \eta, \chi(\eta)) \mathbb{P}_{0}(\mathrm{~d} \eta)}
$$

He then chooses $u_{2}=\psi_{2}(s)$ to maximize his expected pay-off, i.e. such that

$$
\forall u_{2} \in U_{2}, \quad \mathbb{E}^{\mathbb{P}_{2}(\theta \mid s)} J_{2}\left(\theta, \chi(\theta), \psi_{2}(s)\right) \geq \mathbb{E}^{\mathbb{P}_{2}(\theta \mid s)} J_{2}\left(\theta, \chi(\theta), u_{2}\right) .
$$

Assume that player 1 has correctly "guessed" player 2's conjecture $\chi$. He then knows completely how player 2 will construct his decision. i.e., the function $\psi_{2}$. He may choose his own decision $u_{1}=\psi_{1}(\theta)$ to maximize his own expected payoff, i.e. such that,

$$
\forall u_{1} \in U_{1}, \quad \mathbb{E}^{\mathbb{P}_{1}\left(s \mid \theta, \psi_{1}(\theta)\right)} J_{1}\left(\theta, \psi_{1}(\theta), \psi_{2}(s)\right) \geq \mathbb{E}^{\mathbb{P}_{1}\left(s \mid \theta, u_{1}\right)} J_{1}\left(\theta, u_{1}, \psi_{2}(s)\right) .
$$

Definition 1 The pair $\left(\psi_{1}, \psi_{2}\right)$ is a bayesian equilibrium if, in the above construction,

$$
\forall \theta \in \Theta, \quad \psi_{1}(\theta)=\chi(\theta) .
$$

In words: even knowing player 2's conjecture $\chi$, player 1 cannot do better than using that same decision rule.

Remark 1 It is not necessary, in defining a bayesian equilibrium, to introduce a different name for player 2's conjecture $\chi$ and player 1's decision rule $\psi_{1}$, since we want them to coincide. But doing so helps to stress that $\chi$ is part of player 2 's strategy. As usual, a Nash-like equilibrium can be viewed as a fixed point: $\psi_{1}$ is a function $\psi_{1}=\mathcal{F}(\chi)$ and a bayesian equilibrium should satisfy $\mathcal{F}\left(\psi_{1}\right)=\psi_{1}$. At equilibrium, it holds that

$$
\mathbb{P}_{2}(\theta \mid s)=\frac{\mathbb{P}_{1}\left(s \mid \theta, \psi_{1}(\theta)\right) \mathbb{P}_{0}(\theta)}{\int \mathbb{P}_{1}\left(s \mid \eta, \psi_{1}(\eta)\right) \mathbb{P}_{0}(\mathrm{~d} \eta)}
$$


Second form A simplification arises if $J_{2}$ is independent from $u_{1}$. In that case, instead of forming a conjecture on player one's strategy, player 2 may use the signal $s$ to form a conjecture, called a belief, on $\theta$ in the form of a probability law $\mathbb{P}_{2}(\theta \mid s)$. Then, he chooses his strategy $\psi_{2}(s)$ such that,

$$
\forall u_{2} \in U_{2}, \quad \mathbb{E}^{\mathbb{P}_{2}(\theta \mid s)} J_{2}\left(\theta, \psi_{2}(s)\right) \geq \mathbb{E}^{\mathbb{P}_{2}(\theta \mid s)} J_{2}\left(\theta, u_{2}\right) .
$$

If player 1 correctly infers this strategy, he may choose a strategy $\psi_{1}(\theta)$ such that, as previously equations 1 holds.

Definition 2 The system $\psi_{1}, \psi_{2}, \mathbb{P}_{2}$ is a bayesian equilibrium if equation 3 holds.

\subsection{Necessary conditions}

\subsubsection{Types of bayesian equilibria}

We do not attempt to write here first order necessary conditions that a general bayesian equilibrium should satisfy, because we shall only need a particular, and simpler, version of the second form above.

Definition 3 A bayesian equilibrium where $\psi_{1}$ is an invertible function, is called separating (or revealing). Otherwise, the equilibrium is called pooling.

\section{Remark 2}

1. If a separating equilibrium is played, after player 1 has played player 2 is exactly informed of the previously private information $\theta$. The signal is said to be "honest".

2. Our models have two equilibria: a "mixed" equilibrium, separating in a range of the private parameter, pooling in its complement, but essentially behaving as a separating equilibrium, and a trivial pooling equilibrium.

\subsubsection{Separating equilibrium of a pure signaling game}

We consider a pure signaling game $J_{1}: \Theta \times U_{1} \times U_{2} \rightarrow \mathbb{R}, J_{2}: \Theta \times U_{2} \rightarrow \mathbb{R}$, signal $s=u_{1} \in U_{1}$. Let $\varphi: \Theta \rightarrow U_{2}$ give the optimal control $u_{2}$ knowing $\theta$ :

$$
\forall u_{2} \in U_{2}, \quad J_{2}(\theta, \varphi(\theta)) \geq J_{2}\left(\theta, u_{2}\right) .
$$

We have implicitly assumed up to now that the set $U_{1}$ of possible first player's decisions is independent on $\theta$, however, this will not be true in our first model. But this is critical only if an equilibrium strategy lies on the boundary of $U_{1}$. We avoid that problem with the following assumptions: 


\section{Assumptions}

1. $\Theta, U_{1}$ and $U_{2}$ are subsets of $\mathbb{R}$ or $\mathbb{R}$ itself, ${ }^{2}$

2. $J_{1} \in \mathcal{C}^{1}, J_{2} \in \mathcal{C}^{2}$,

3. $J_{2}$ is locally strictly concave in $u_{2}$ in a neighborhood of its maximum and

$$
\frac{\partial^{2} J_{2}}{\partial u_{2}^{2}}(\theta, \varphi(\theta))<0
$$

4. There exists a bayesian equilibrium with, for all $\theta, \psi_{1}(\theta)$ in the interior of $U_{1}$ and $\psi_{2} \circ \psi_{1}(\theta)$ in the interior of $U_{2} \cdot\left(^{3}\right)$

Handicap principle Under these assumptions, the optimality of $\psi_{1}$ implies

$$
\frac{\mathrm{d} J_{1}\left(\theta, u_{1}, \psi_{2}\left(u_{1}\right)\right)}{\mathrm{d} u_{1}}=\left.\left(\frac{\partial J_{1}}{\partial u_{1}}+\frac{\partial J_{1}}{\partial u_{2}} \psi_{2}^{\prime}\right)\right|_{\left(u_{1}, u_{2}\right)=\left(\psi_{1}(\theta), \psi_{2}\left(\psi_{1}(\theta)\right)\right)}=0 .
$$

Hence, the direct effect of $u_{1}$ on $J_{1}$ and its indirect effect through player two's response are necessarily of opposite signs.

Much of the literature fails to define precisely what is called "handicap principle". We use this phrase for the following proposition, which is a corollary of the above essentially trivial remark:

Proposition 1 If we assume that the aim of the signal is to obtain a favorable response from the other player, then, under assumptions 1 to 4 above, its direct effect $\partial J_{1} / \partial u_{1}$ has to be negative at equilibrium.

\subsubsection{Computing the equilibrium strategies}

According to the hypothesis that the optimal decisions are interior decisions, we have

$$
\frac{\partial J_{2}}{\partial u_{2}}(\theta, \varphi(\theta))=0
$$

and, by the implicit function theorem,

$$
\varphi^{\prime}(\theta)=\frac{\mathrm{d} \varphi}{\mathrm{d} \theta}=-\frac{\partial^{2} J_{2}}{\partial u_{2} \partial \theta}(\theta, \varphi(\theta))\left(\frac{\partial^{2} J_{2}}{\partial u_{2}^{2}}(\theta, \varphi(\theta))\right)^{-1} .
$$

\footnotetext{
${ }^{2}$ In our model, the females' decision is a time function.

${ }^{3}$ Note that this assumption is violated in the case of "index signals" [9] to be discussed, as well as in our trivial equilibrium.
} 
If the equilibrium is separating, the optimal $\psi_{2}$ is

$$
\psi_{2}\left(u_{1}\right)=\varphi\left(\psi_{1}^{-1}\left(u_{1}\right)\right),
$$

and hence, according to the chain rule

$$
\psi_{2}^{\prime}\left(u_{1}\right)=\varphi^{\prime}\left(\psi_{1}^{-1}\left(u_{1}\right)\right) \frac{1}{\psi_{1}^{\prime}(\theta)} .
$$

Substituting this and equation (6) in (5), one obtains that for $u_{1}=\psi_{1}(\theta)$,

$$
\begin{aligned}
0 & =\frac{\mathrm{d} J_{1}\left(\theta, u_{1}, \psi_{2}\left(u_{1}\right)\right)}{\mathrm{d} u_{1}} \\
& =\frac{\partial J_{1}}{\partial u_{1}}\left(\theta, u_{1}, \psi_{2}\left(u_{1}\right)\right)-\frac{\partial J_{1}}{\partial u_{2}}\left(\theta, u_{1}, \psi_{2}\left(u_{1}\right)\right) \frac{\frac{\partial^{2} J_{2}}{\partial u_{2} \partial \theta}\left(\theta, \psi_{2}\left(u_{1}\right)\right)}{\frac{\partial^{2} J_{2}}{\partial u_{2}^{2}}\left(\theta, \psi_{2}\left(u_{1}\right)\right)} \frac{1}{\psi_{1}^{\prime}(\theta)},
\end{aligned}
$$

hence

$$
\psi_{1}^{\prime}(\theta)=\frac{\frac{\partial J_{1}\left(\theta, \psi_{1}(\theta), \psi_{2}\left(\psi_{1}(\theta)\right)\right)}{\partial u_{2}}}{\frac{\partial J_{1}\left(\theta, \psi_{1}(\theta), \psi_{2}\left(\psi_{1}(\theta)\right)\right)}{\partial u_{1}}} \frac{\frac{\partial^{2} J_{2}}{\partial u_{2} \partial \theta}\left(\theta, \psi_{2}\left(u_{1}\right)\right)}{\frac{\partial^{2} J_{2}}{\partial u_{2}^{2}}\left(\theta, \psi_{2}\left(u_{1}\right)\right)} .
$$

The point is that, as a rule, the first order necessary conditions yield a differential equation on $\psi_{1}$. There remains to choose an initial condition for it. As $u_{1}$ is costly, the best possible initial condition is the smallest possible one within the constraints of the problem at hand.

Finally, notice that we know that, under our assumptions, both $\partial J_{1} / \partial u_{1}$ and $\partial^{2} J_{2} / \partial u_{2}^{2}$ have to be negative. Assuming that $\partial J_{1} / \partial u_{2}$ is positive (hence also $\psi_{2}^{\prime}$ ), then, it follows from equation (7) that the equilibrium signal is indeed monotonously increasing, hence honest, as well as the receiver's response, if, at equilibrium

$$
\frac{\partial^{2} J_{2}}{\partial u_{2} \partial \theta}\left(\theta, \psi_{2}(\theta)\right)>0
$$

This condition bears on the receiver's marginal sensitivities. It looks different from Getty [5] equation (5), but only because Getty writes his condition in terms of the signaller's resulting fitness taking into account the receiver's response $(P(a)$ in his notation). The receiver's criterion is implicit in his model, while it is explicit here.

\section{Two models of sexual selection}

\subsection{The framework}

Most previous studies have modelled fitness as the product of survivorship and mating success, where survivorship is a lifetime measure of the expected number of 
breeding attempts. ([15]). Let therefore the quality of an individual male denote his survivorship, more precisely the expected number of breeding seasons he will live through, in the absence of the negative impact of the secondary sexual characters used as a signal. The qualities of male individuals are distributed according to a probability law $\mathbb{P}_{0}$, that we shall quickly assume uniform.

Quality will be denoted by $q \in\left(q_{0}, q_{1}\right]$. The latter quantity can indeed be bounded above by $q_{1}=1$ for instance, which means that the species considered live through a single breeding season (annual or semelparous species) [7], or by a larger $q_{1}$ for iteroparous species enjoying as a rule several reproductive bouts. Grafen also considered that the expected number of breeding seasons can be bounded below by a strictly positive value $q_{0}>0$. It is obvious that from the female point of view, $q$ cannot be zero. Yet, it could definitely be arbitrarily close to zero, implying $q_{0}=0$, and we will see that this parameter has a great influence on Grafen's model.

To show why $q_{0}$ has such an influence on Grafen's model, we will compare two closely related models. We call one the multiplicative signaling cost model, since in this model, the measure of the signal $\sigma \in[0,1]$ is the factor by which the survivorship $L_{\sigma^{7}}$ of a male is reduced: $L_{\sigma^{7}}=q(1-\sigma)$. This model will be shown to be akin to Grafen's, as opposed to the additive signaling cost model, also alluded to in [5] who attributes it to [14], in which the measure of the signal $s>0$ is the quantity by which survivorship is reduced: $L_{\mathrm{O}^{x}}(q, s)=q-s$. In this model, males have negative fitness as soon as they signal greater than $q$. These two models could be used to compare "index" (unfakeable) signaling with strategic (unconstrained by quality) signaling [9]. Yet, we will focus on interior equilibria on the basis of local stability conditions, so this dichotomy will not be relevant in this study. As a last remark, we stress that in each model, contrary to [10] for instance, males vary by their quality only; males of same quality "pay" the same amount for the same signal.

In both cases, the expected number of successful matings per breeding season, or mating success rate, is denoted $N_{m}$. It is a function of the signal, $\sigma$ or $s$, and of how females behave in response to it. To evaluate that number, we assume that the breeding season is of length $T$ each year; $t$ is the time elapsed since the beginning of the breeding season. We however neglect the event of a male dying during that season. Male's fitness therefore is $F_{\sigma^{7}}=L_{\sigma^{7}} N_{m}$.

For each model, we shall consider two extreme cases, depending on whether the limiting factor for the males, beyond being accepted by females, is their (constant) potential reproduction rate (P.R.R.) $\mu$, or the scarcity of females, measured by an (increasing) operating sex ratio (O.S.R.) $\omega(t)$.

Each female meets males in a Poisson process of intensity $\lambda$. She observes the signal exactly, and chooses a probability $m=\varphi_{\odot}(\sigma)$ in the first model, $m=\psi_{\odot}(s)$ 
in the second, to accept to mate. Her fitness will be taken to be the quality of the male she will mate with, its expectation $Q_{0}=F_{\text {o }}$ being the same for all females. After mating, she leaves the pool of females available for mating.

It will be convenient to use all along the following dimensionless parameters

$$
k=\frac{2}{\lambda T}, \quad \tau=\frac{T-t}{T},
$$

where $\lambda T$ is the mean number of males encounter by females in one breeding season.

\subsection{Females' behavior}

In this section, we use the same notation $s$ for $s$ or $\sigma$.

\subsubsection{Females' strategy}

According to the earlier introduction to signaling theory, we let $\chi(q)$ be the conjecture made by the females about the males' strategy, i.e. $\chi^{-1}(s)$, be her estimate of a male's quality given his signal $s$.

Let $Q(t)$ be the expected quality of the male she will mate with for a female that has not yet mated at time $t$. In the forthcoming infinitesimal time interval $\mathrm{d} t$, she will meet a male with a probability $\lambda \mathrm{d} t$, whose quality $q$ is distributed according to the probability law $\mathbb{P}_{0}$, and displaying an a priori unknown signal $s$, and she will accept to mate with him with a probability $m$, her choice, which may depend on $t$ and $s$. We therefore get

$$
Q(t)=\mathbb{E}[\lambda \mathrm{d} t m q+(1-\lambda \mathrm{d} t m) Q(t+\mathrm{d} t)] .
$$

In the limit as $\mathrm{d} t \rightarrow 0$, this yields

$$
\frac{\mathrm{d} Q}{\mathrm{~d} t}+\lambda \mathbb{E}[m(q-Q(t))]=0, \quad Q(T)=0 .
$$

(In this equation, $m$ is allowed to be a random variable measurable on the sigma algebra generated by q.) It follows from the theorem of comparison of the solutions of ordinary differential equations that to maximize $Q(0)$, the females should maximize $\mathbb{E}[m(q-Q)]$, i.e. choose

$$
m= \begin{cases}0 & \text { if } q<Q(t) \\ 1 & \text { if } q \geq Q(t)\end{cases}
$$


However, they observe neither $q$ nor $Q$. They will have to rely on $q=\chi^{-1}(s)$, and choose the threshold strategy

$$
m=\psi_{\uparrow}(s ; t):= \begin{cases}0 & \text { if } s<\theta(t), \\ 1 & \text { if } s \geq \theta(t) .\end{cases}
$$

with

$$
\theta(t)=\chi(Q(t)),
$$

where $Q(t)$ is calculated as the solution of the differential equation obtained on the basis that $s=\chi(q)$, i.e. that $q$ is exactly recovered from $s$. Let, for any $X \in \mathbb{R}$,

$$
[X]_{+}= \begin{cases}0 & \text { if } X \leq 0 \\ X & \text { if } X>0\end{cases}
$$

The differential equation for $Q(t)$ reads:

$$
\frac{\mathrm{d} Q}{\mathrm{~d} t}+\lambda \mathbb{E}[q-Q(t)]_{+}=0, \quad Q(T)=0,
$$

or, equivalently

$$
\frac{\mathrm{d} Q}{\mathrm{~d} t}+\lambda \mathbb{E}[q-Q(t) \mid q \geq Q(t)] \mathbb{P}_{0}\left(\left[Q(t), q_{1}\right]\right)=0, \quad Q(T)=0 .
$$

To carry the calculations further, we make the following assumption:

Assumption The probability law $\mathbb{P}_{0}$ is uniform over $\left[q_{0}, q_{1}\right]$.

To go further, we need to discuss whether $Q(t)$ is smaller or larger than $q_{0}$.

1. If $Q \leq q_{0}$, then

$$
\mathbb{E}[q-Q]_{+}=\frac{q_{1}+q_{0}}{2}-Q,
$$

hence

$$
\frac{\mathrm{d} Q}{\mathrm{~d} t}=-\lambda\left(\frac{q_{1}+q_{0}}{2}-Q\right) \quad Q(T)=0,
$$

and

$$
Q(t)=\frac{q_{0}+q_{1}}{2}\left(1-\mathrm{e}^{-\lambda(T-t)}\right)
$$

from the time $T_{0}$ at which $Q\left(T_{0}\right)=q_{0}$, i.e.

$$
\mathrm{e}^{-\lambda\left(T-T_{0}\right)}=\frac{q_{1}-q_{0}}{q_{1}+q_{0}},
$$



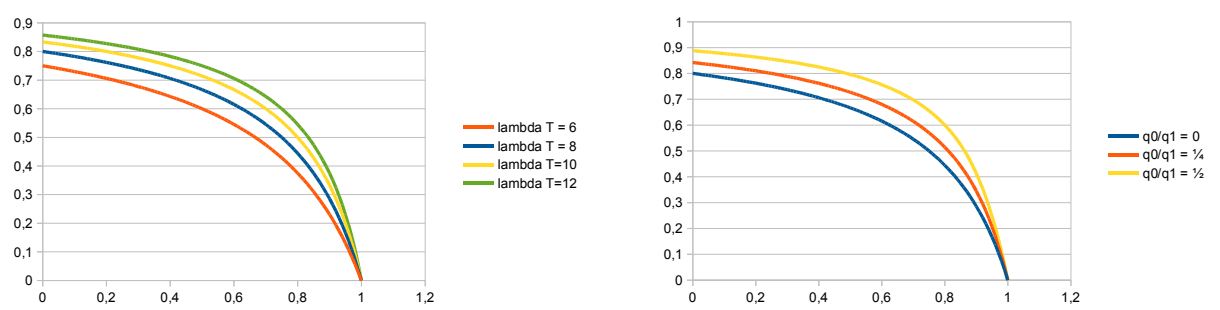

Figure 1: Curves $Q(t) / q_{1}$, to the left, for $q_{0}=0$, with various values of $\lambda T$, to the right, for $\lambda T=8$ and different values of $q_{0} / q_{1}$.

up to time $T$. We shall use the notation

$$
\tau_{0}:=\frac{T-T_{0}}{T}=\frac{k}{2} \ln \frac{q_{1}+q_{0}}{q_{1}-q_{0}} .
$$

2. If $Q \geq q_{0}$, then

$$
\mathbb{E}[q-Q]_{+}=\frac{1}{q_{1}-q_{0}} \int_{Q}^{q_{1}}(q-Q) \mathrm{d} q=\frac{\left(q_{1}-Q\right)^{2}}{2\left(q_{1}-q_{0}\right)},
$$

hence

$$
\frac{\mathrm{d} Q}{\mathrm{~d} t}=-\lambda \frac{\left(q_{1}-Q\right)^{2}}{2\left(q_{1}-q_{0}\right)}, \quad Q\left(T_{0}\right)=q_{0}
$$

Integrating this last equation we summarize:

$$
Q(t)= \begin{cases}\frac{k \frac{q_{0}}{q_{1}} T+T_{0}-t}{k T+T_{0}-t} & \text { if } t \leq T_{0}, \\ \frac{q_{0}+q_{1}}{2}\left(1-\mathrm{e}^{-\lambda(T-t)}\right) & \text { if } t \geq T_{0} .\end{cases}
$$

Notice that

$$
\forall t \in[0, T], \quad Q(t) \leq Q_{0}:=q_{1} \frac{k \frac{q_{0}}{q_{1}} T+T_{0}}{k T+T_{0}} .
$$

Equations (9), (10), (11), and (13) define the females' strategy in terms of their conjecture $\chi$ about the males' strategy. In figure 1, we have plotted curves of $Q(t)$ against $t / T$. 


\subsubsection{Consequences for the males}

A male displaying a signal $s$ mates only when $Q(t) \leq \chi^{-1}(s)$, i.e. when $t \geq t_{m}$ with

$$
\tau_{m}:=\frac{T-t_{m}}{T}= \begin{cases}1 & \text { if } \chi^{-1}(s) \geq Q_{0}, \\ \tau_{0}+k \frac{\chi^{-1}(s)-q_{0}}{q_{1}-\chi^{-1}(s)} & \text { if } \chi^{-1}(s) \leq Q_{0} .\end{cases}
$$

It will be useful in the sequel to note that, for $q<Q_{0}$,

$$
\frac{\mathrm{d} \tau_{m}}{\mathrm{~d} s}=\tau_{m}^{\prime}=k\left(1-\frac{q_{0}}{q_{1}}\right) \frac{q_{1}}{\chi^{\prime}\left(\chi^{-1}(s)\right)\left(q_{1}-\chi^{-1}(s)\right)^{2}} .
$$

Another consequence is that the number of females available for mating decreases during the breeding season. At time $t$, there are $n_{q}(t)$ unmated females. Let $N_{\rho}=\mathbb{E} n_{\text {o }}$. During an infinitesimal time interval $\mathrm{d} t$, an expected number $N_{\varphi}(t) \lambda \mathrm{d} t$ of females meet a male, and a proportion $\left(q_{1}-Q(t)\right) /\left(q_{1}-q_{0}\right)$ of these males are accepted as mates, as long as $Q<q_{0}$, i.e. for $t<T_{0}$, and all are accepted once $t \geq T_{0}$. The females that have accepted a mate leave the pool of available females. Therefore we have for the expected number of available females

$$
\frac{\mathrm{d} N_{\varphi}}{\mathrm{d} t}= \begin{cases}-N_{\varphi} \lambda \frac{q_{1}-Q(t)}{q_{1}-q_{0}}=-N_{\odot} \frac{2}{k T+T_{0}-t} & \text { if } t<T_{0}, \\ -\lambda N_{\varphi} & \text { if } t \geq T_{0} .\end{cases}
$$

and therefore

$$
N_{\varphi}(t)= \begin{cases}N_{\varphi}(0)\left(\frac{k T+T_{0}-t}{k T+T_{0}}\right)^{2} & \text { if } t<T_{0}, \\ N_{\varphi}(0)\left(\frac{k T}{k T+T_{0}}\right)^{2} \frac{q_{1}-q_{0}}{q_{1}+q_{0}} \mathrm{e}^{-\lambda(t-T)} & \text { if } t \geq T_{0} .\end{cases}
$$

\subsection{Males' strategy: multiplicative signaling cost}

\subsubsection{An abstract derivation}

Notation We investigate our first model, i.e. one where the signal is $\sigma \in[0,1]$ and the survivorship

$$
L_{\mathrm{O}^{\top}}=q(1-\sigma), \quad \sigma \in[0,1] .
$$

We let $q \in\left[q_{0}, q_{1}\right]$ with $q_{0}>0$.

The females' strategy is as above, and therefore $t_{m}(s)$ is given by equation (15) and $N_{\varphi}(t)$ by (17). We denote $\sigma=\varphi_{\sigma^{\prime}}(q)$ the males' strategy in this model. 
We use the notation

$$
\begin{aligned}
F_{\sigma^{7}}(q, \sigma, m(\cdot)) & =L_{\sigma^{7}}(q, \sigma) N_{m}(m(\cdot)), \\
\widehat{F}_{\sigma^{7}}(q, \sigma) & :=F_{\sigma^{7}}\left(q, \sigma, \varphi_{\varphi}(\sigma ; \cdot)\right)=L_{\sigma^{7}}(q, \sigma) N_{m}\left(\varphi_{q}(\sigma)\right),
\end{aligned}
$$

and

$$
\varphi_{\uparrow}(\sigma, t)=\hat{\varphi}_{q}\left(\chi^{-1}(\sigma), t\right), \quad \widehat{N}_{m}(q):=N_{m}\left(\hat{\varphi}_{q}(q, \cdot)\right)
$$

so that

$$
\widehat{F}_{\sigma^{7}}(q, \sigma)=L_{\sigma^{7}}(q, \sigma) \widehat{N}_{m}\left(\chi^{-1}(\sigma)\right) \text {. }
$$

Deriving $\varphi_{\sigma^{7}}$ and $F_{\sigma^{x}} \quad$ According to section 2, we derive $\varphi_{\sigma^{x}}(q)$ by differentiating $\widehat{F}_{\sigma^{\prime}}$ with respect to $\sigma$ and equating to zero. Using $\left(\chi^{-1}\right)^{\prime}(\sigma)=1 / \chi^{\prime}\left(\chi^{-1}(\sigma)\right)$, this yields, for $q<Q_{0}$ :

$$
\frac{\partial \widehat{F}_{\sigma^{7}}(q, \sigma)}{\partial \sigma}=-q \widehat{N}_{m}\left(\chi^{-1}(\sigma)\right)+q(1-\sigma) \frac{\widehat{N}_{m}^{\prime}\left(\chi^{-1}(\sigma)\right)}{\chi^{\prime}\left(\chi^{-1}(\sigma)\right)}
$$

Replacing $\chi^{-1}(\sigma)$ by $q, \chi$ by $\varphi_{\sigma^{x}}$ and $\sigma$ by $\varphi_{\sigma^{7}}(q)$, and equating to zero, we obtain

$$
0=-\widehat{N}_{m}(q)+\left(1-\varphi_{\sigma^{7}}(q)\right) \frac{\widehat{N}_{m}^{\prime}(q)}{\varphi_{\sigma^{7}}^{\prime}(q)},
$$

or, equivalently

$$
\frac{\varphi_{\mathrm{O}^{7}}^{\prime}(q)}{1-\varphi_{\mathrm{O}^{7}}(q)}=\frac{\widehat{N}_{m}^{\prime}(q)}{\widehat{N}_{m}(q)} .
$$

Using $\varphi_{\mathrm{O}^{7}}(0)=0$, it follows immediately that

$$
\left(1-\varphi_{\sigma^{7}}(q)\right) \widehat{N}_{m}(q)=\widehat{N}_{m}\left(q_{0}\right), \quad \widehat{F}_{\sigma^{7}}\left(q, \varphi_{\sigma^{7}}(q)\right)=q \widehat{N}_{m}\left(q_{0}\right) .
$$

When $q \geq Q_{0}$, there is no incentive for the males to signal more than for $q=Q_{0}$, since anyway, with this signal $\tau_{m}=1$. We let thus $\varphi_{\mathrm{O}^{7}}(q)=\varphi_{\mathrm{O}^{7}}\left(Q_{0}\right)$, $\widehat{N}_{m}(q)=\widehat{N}_{m}\left(Q_{0}\right)$, so that $\left(1-\varphi_{\sigma^{7}}(q)\right) \widehat{N}_{m}(q)=\left(1-\varphi_{\sigma^{7}}\left(Q_{0}\right)\right) \widehat{N}_{m}\left(Q_{0}\right)=$ $\widehat{N}_{m}\left(q_{0}\right)$, and equation (21) still holds.

\subsubsection{Equilibrium solutions of two variants}

P.R.R. as the limiting factor

In this first simpler variant, we deviate somewhat from the stated hypothesis that males can mate an arbitrary number of times. We assume that the number of available females remains large and the meeting rate high, and that, once accepted by 
females, the limit on males' number of matings is their potential reproduction rate $\mu$, limited, say, by the time it takes to produce sperm. Il this model, $\lambda$ is the rate of females meeting a male ready for mating. In this case, we simply have $N_{m}(\sigma)=\mu T \tau_{m}(\sigma)$. Hence

$$
1-\varphi_{\sigma^{7}}(q)=\frac{\widehat{N}_{m}\left(q_{0}\right)}{\widehat{N}_{m}(q)}=\frac{\tau_{0}\left(q_{1}-q_{0}\right)}{\tau_{0}\left(q_{1}-q\right)+k\left(q-q_{0}\right)},
$$

or

$$
\varphi_{\mathrm{O}^{\Upsilon}}(q)=\frac{k\left(q-q_{0}\right)}{\tau_{0}\left(q_{1}-q\right)+k\left(q-q_{0}\right)},
$$

and

$$
\widehat{F}_{\sigma^{\top}}\left(q, \varphi_{\sigma^{\top}}(q)\right)=q \mu T \tau_{0} .
$$

Remember that $\tau_{0}$ is given by equation (12), and is zero if $q_{0}=0$. Therefore in that case, we get $\varphi_{\mathrm{O}^{7}}(q)=1$ and $\widehat{F}_{\mathrm{O}^{\top}}=0$ for all $q>0$, an absurd conclusion. We must therefore assume $q_{0}>0$. We shall come back to this point in the discussion.

Scarcity of the females as the limiting factor

In this variant, we come back to the hypothesis that the P.R.R. of males is large, and that the only limit on their number of matings, once accepted by females, is the increasing scarcity of mating opportunities while the breeding season goes on. We ignore explicit infighting between males to get access to females, which is a reasonable assumption as long as the initial operating sex ratio is low enough that males seldom meet each other.

The population under consideration is assumed to be in a steady state, with $N_{\sigma^{7}}$ males and $N_{\phi}(0)$ females at the start of each breeding season. $N_{\sigma^{r}}$ being constant, so is the rate $\lambda$ at which females meet males. Each useful meeting involves a male and a female. Hence, the process of males meeting an available female is Poisson with an intensity $\mu(t)$ such that $N_{\bigcirc^{\top}} \mu(t)=n_{\odot}(t) \lambda$. Therefore, writing $\mu(0)=\mu_{0}$,

$$
\mu(t)=\lambda n_{\varphi}(t) / N_{\sigma^{r}}=\mu_{0} n_{\uparrow}(t) / N_{\varphi}(0) .
$$

We can now estimate the expected mating rate using (17) (and Fubini's theorem):

$$
\begin{aligned}
N_{m} & =\mathbb{E} \int_{t_{m}}^{T} \mu(s) \mathrm{d} s=\frac{\mu_{0}}{N_{\varphi}(0)} \int_{t_{m}}^{T} N_{\varphi}(s) \mathrm{d} s \\
& =\frac{\mu_{0}}{N_{\varphi}(0)}\left[\int_{t_{m}}^{T_{0}} N_{\varphi}(s) \mathrm{d} s+\int_{T_{0}}^{T} N_{\varphi}(s) \mathrm{d} s\right] \\
& =\frac{\mu_{0} k^{3} T^{3}}{\left(k T+T_{0}\right)^{2}}\left[\frac{q_{0}}{q_{1}+q_{0}}+\frac{1}{3}\left(1+\frac{\tau_{m}-\tau_{0}}{k}\right)^{3}-\frac{1}{3}\right] .
\end{aligned}
$$


We use this with equation (15) to get

$$
\widehat{N}_{m}(q)=\frac{K}{3}\left[\frac{3 q_{0}}{q_{1}+q_{0}}+\left(\frac{q_{1}-q_{0}}{q_{1}-q}\right)^{3}-1\right]
$$

with

$$
K:=\frac{\mu_{0} T^{3} k^{3}}{\left(k T+T_{0}\right)^{2}}
$$

and finally

$$
\begin{aligned}
\varphi_{\sigma^{7}}(q) & =\frac{\left(q_{1}-q_{0}\right)^{3}-\left(q_{1}-q\right)^{3}}{\left(q_{1}-q_{0}\right)^{3}-\frac{q_{1}-2 q_{0}}{q_{1}+q_{0}}\left(q_{1}-q\right)^{3}}, \\
\widehat{F}_{\sigma^{7}}\left(q, \varphi_{\sigma^{7}}(q)\right) & =K \frac{q_{0}}{q_{1}+q_{0}} q .
\end{aligned}
$$

Note that this model again displays the feature that if $q_{0}=0$, then $\varphi_{\mathrm{O}^{7}}(q)=1$ and $\widehat{F}_{\mathrm{O}^{7}}=0$ for all $q>0$, an absurd conclusion.

In figure 2, we have plotted the strategy $\varphi_{0^{7}}$, the relative survivorship $L_{\sigma^{7}} / q_{1}$ and the overall fitness $F_{\sigma^{T}} / q_{1}$ of males for $\lambda T=8$, and for different values of $q_{0} / q_{1}$ in the case of a limiting availability of females. We have included $q_{0} / q_{1}=.5$ for clarity, but we consider this value beyond a realistic model. See the foregoing discussion.

\subsection{Males' strategy: additive signaling cost}

In this section, we investigate a model somewhat more different from Grafen's than the first one, in that we assume that, denoting $s$ the signal,

$$
L_{\mathrm{O}^{T}}=q-s, \quad s \in[0, q] .
$$

In this model, we will see that it is possible to take $q_{0}=0$, and hence $T_{0}=T$, $\tau_{0}=0$, and $Q_{0}=q_{1} /(1+k)$. We denote $\varphi_{0^{7}}(s)$ the males' strategy, and we use the notation (19) mutatis mutandis.

\subsubsection{Limiting P.R.R.}

As for the first model, we first consider the case where $\mu$ is constant. The expected number of matings in any season is $\mu\left(T-t_{m}\right)$, and therefore the males' fitness is

$$
\widehat{F}_{\sigma^{7}}(q, s)=\mu T(q-s) \tau_{m}(s) .
$$



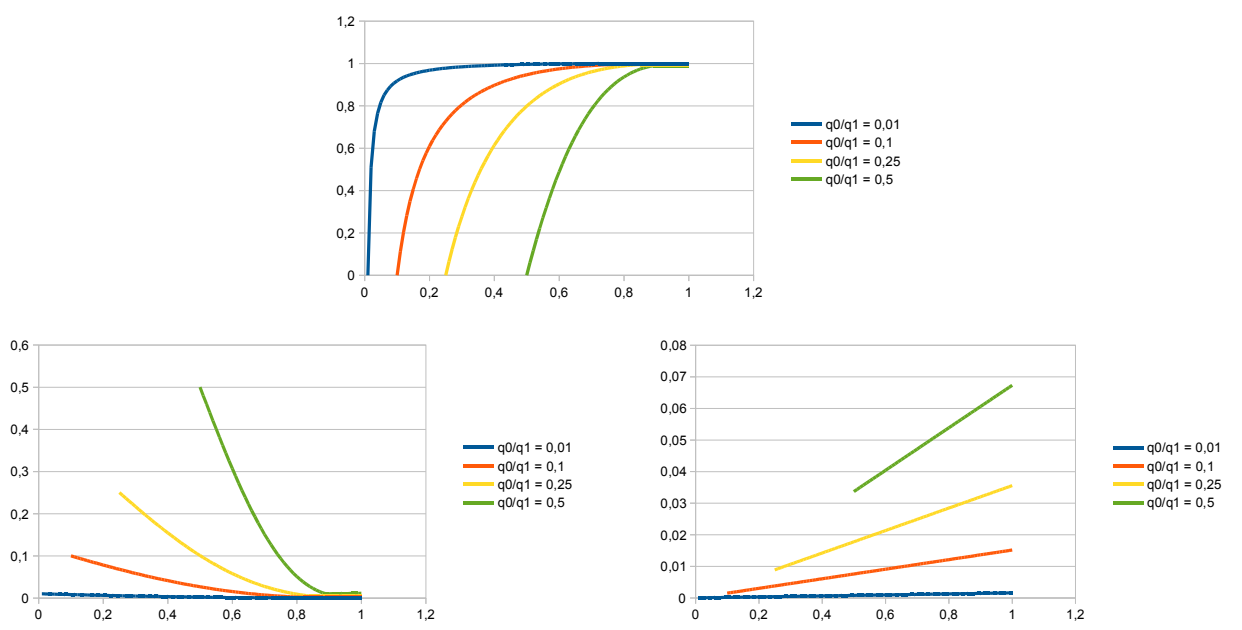

Figure 2: Multiplicative model: Males' strategy $\varphi_{\sigma^{T}}$ (top), relative survivorship $L_{\mathrm{O}^{\mathrm{T}}} / q_{1}$ (bottom left) and overall fitness $F_{\mathrm{O}^{\mathrm{T}}} / q_{1}$ (bottom right) as a function of $q / q_{1}$, and for $\lambda T=8$ and several values of $q_{0} / q_{1}$.

Clearly, for $\chi^{-1}(s) \geq q_{1} /(1+k), F_{\sigma^{7}}$ is strictly decreasing in $s$, so that it is useless for the males to use a signal larger than $\chi\left(q_{1} / 1+k\right)$. For smaller $s$, we have, using the fact that $\left(\chi^{-1}\right)^{\prime}=\left(\chi^{\prime}\right)^{-1}$ :

$$
\frac{1}{\mu T k} \frac{\partial \widehat{F}_{\sigma^{\top}}}{\partial s}=-\frac{\chi^{-1}(s)}{q_{1}-\chi^{-1}(s)}+\frac{\chi^{-1}(s)-s}{\left(q_{1}-\chi^{-1}(s)\right) \chi^{\prime}\left(\chi^{-1}(s)\right)}
$$

Now, at equilibrium, $s=\psi_{o^{7}}(q)=\chi(q)$ so that also $\chi^{-1}(s)=q$. Equating the above derivative to zero, we get

$$
\psi_{\sigma^{\Im}}^{\prime}(q)=q_{1} \frac{q-\psi_{\sigma^{7}}(q)}{q\left(q_{1}-q\right)}, \quad \psi_{\sigma^{7}}(0)=0 .
$$

We initialize this differential equation at $\psi_{\sigma^{7}}(0)=0$ in order to keep $s$ as small as compatible with the equation.

It turns out that equation (24) has a closed form solution $\widetilde{\Psi}(q)$ :

$$
\begin{aligned}
& \widetilde{\Psi}(q)=q_{1}\left[1+\frac{q_{1}-q}{q} \ln \left(1-\frac{q}{q_{1}}\right)\right] \text { if } q \neq 0, \\
& \widetilde{\Psi}(0)=0 .
\end{aligned}
$$

It takes a power expansion in the neighborhood of $q=0$ to check that indeed, this $\widetilde{\Psi}$ is continuous at 0 , and also $\widetilde{\Psi}^{\prime}(0)=1 / 2$ and $\widetilde{\Psi}^{\prime \prime}(0)=1 / 3$. 
We therefore end up with definition (25) and

$$
\psi_{\sigma^{7}}(q)= \begin{cases}\widetilde{\Psi}(q) & \text { if } q \leq \frac{q_{1}}{1+k}, \\ \widetilde{\Psi}\left(\frac{q_{1}}{1+k}\right) & \text { if } q \geq \frac{q_{1}}{1+k} .\end{cases}
$$

It remains to substitute this $\psi_{0^{r}}$ to $\chi$ in equation (10) to have the complete bayesian equilibrium of the signaling game, which is pooling in the upper range $q \geq q_{1} /(1+k)$, and separating for smaller $q$.

\subsubsection{Scarcity of females as limiting factor}

We investigate now the full model with an essentially unlimited P.R.R. of the males. Their meeting rate of females is therefore given by (22). Using (17) with $T_{0}=T$, it follows that

$$
\mathbb{E} \mu(t)=\mu_{0}\left(\frac{k+\tau}{k+1}\right)^{2} .
$$

We use this in evaluating the expected number of matings of a male in one season as

$$
N_{m}=\int_{t_{m}}^{T} \mathbb{E} \mu(t) \mathrm{d} t=\mu_{0} T \int_{0}^{\tau_{m}}\left(\frac{k+\tau}{k+1}\right)^{2} \mathrm{~d} \tau=\frac{\mu_{0} T}{(k+1)^{2}}\left(k^{2} \tau_{m}+k \tau_{m}^{2}+\frac{1}{3} \tau_{m}^{3}\right)
$$

and,

$$
\widehat{F}_{\sigma^{\top}}(q, s)=\frac{\mu_{0} T}{(k+1)^{2}}(q-s)\left(k^{2} \tau_{m}(s)+k \tau_{m}(s)^{2}+\frac{1}{3} \tau_{m}(s)^{3}\right),
$$

where $\tau_{m}$ is still given by (15).

We may again notice that for $q>q_{1} /(1+k)$, it is useless for the males to use a signal greater than for $q=q_{1} /(1+k)$. For lower $q$, we differentiate $\widehat{F}_{\sigma^{7}}$ with respect to $s$, and equate to zero, replacing $\chi^{-1}(s)$ by $q$ and $s$ by $\psi_{\sigma^{7}}(q)$, to get

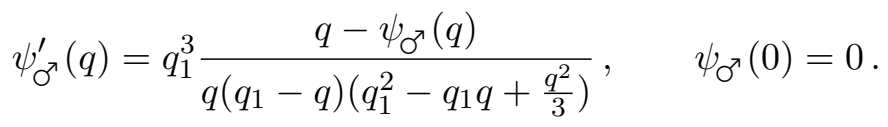

Moreover, this stationary point is indeed a maximum, since $\widehat{F}_{\sigma^{7}}(q, 0)=\widehat{F}_{\sigma^{7}}(q, q)=$ 0 , and $\widehat{F}_{\sigma^{7}}(q, \cdot)$ is positive and differentiable for $s \in(0, q)$. Thus, the stationary point being unique, it necessarily is a maximum.

Unexpectedly, the differential equation (28) has a closed form, and even rational, solution. Since a direct check that formula (29) does satisfy the equation is not 
so easy, we provide a direct derivation in the appendix. This closed form solution of the differential equation (28) is

$$
\Psi(q)=q_{1} \frac{27 q_{1}^{5} q+18 q_{1}^{4} q^{2}+9 q_{1}^{3} q^{3}+3 q_{1}^{2} q^{4}-q^{6}}{2\left(27 q_{1}^{6}+q^{6}\right)}
$$

One may notice that, on the one hand, both (24) and (28) imply that $\psi_{\mathrm{O}^{7}}^{\prime}(0)=1 / 2$, but the second one yields $\psi_{O^{7}}^{\prime \prime}(0)=2 / 3$, so that for small $q, \Psi(q)$ is "above" $\widetilde{\Psi}(q)$. On the other hand, for $s, q \in\left(0, q_{1}\right)$,

$$
q_{1} \frac{q-s}{q\left(q_{1}-q\right)} \leq q_{1}^{3} \frac{q-s}{q\left(q_{1}-q\right)\left(q_{1}^{2}-q_{1} q+\frac{q^{2}}{2}\right)}
$$

so that, by a standard comparison principle, for all $q$ in $\left[0, q_{1}\right], \widetilde{\Psi}(q) \leq \Psi(q)$. But since $\psi_{\sigma^{7}}(q)$ must remain less than $q$, it follows that necessarily, $\Psi\left(q_{1}\right)=q_{1}$, which is indeed satisfied by (29).

Finally, the equilibrium strategies of this model are given by equations (9), (13), (10) where we substitute $\psi_{\sigma^{r}}$ to $\chi,(29)$, and

$$
\psi_{\sigma^{(}}(q)= \begin{cases}\Psi(q) & \text { if } q \leq \frac{q_{1}}{k+1}, \\ \Psi\left(\frac{q_{1}}{k+1}\right) & \text { if } q \geq \frac{q_{1}}{k+1} .\end{cases}
$$

In figure 3, we have plotted the strategy $\psi_{\mathrm{O}^{\top}} / q_{1}$, the relative survivorship $L_{\mathrm{O}^{\top}} / q_{1}$, and the overall fitness $F_{\sigma^{7}} / q_{1}$ of males against $q / q_{1}$, for $\lambda T=8$, for the case of a limiting P.R.R. (fixed $\mu$ ) or a limiting availability of females (variable $\mu$ ).

\subsection{Discussion}

\subsubsection{General synthesis}

The paradigm of bayesian equilibrium is a natural one to deal with the role of sexually selected characters, this independently of whether the underlying mechanism is one of direct benefit, as in [8], or indirect benefit as allowed by our model. Indeed, what game theory teaches us is that, if these secondary sexual characters have for unique, or main, role in the evolutionary process, to transmit information about their bearer's quality to the individuals of the other sex, and if they are not physically constrained by that quality (see [9]), then at equilibrium it must be that the direct effect of these characters is to lower the viability of their bearer, trading viability for greater sexual success. And the nature of a (separating) Bayesian equilibrium is that the possibility of profitable cheating has been defined out. 

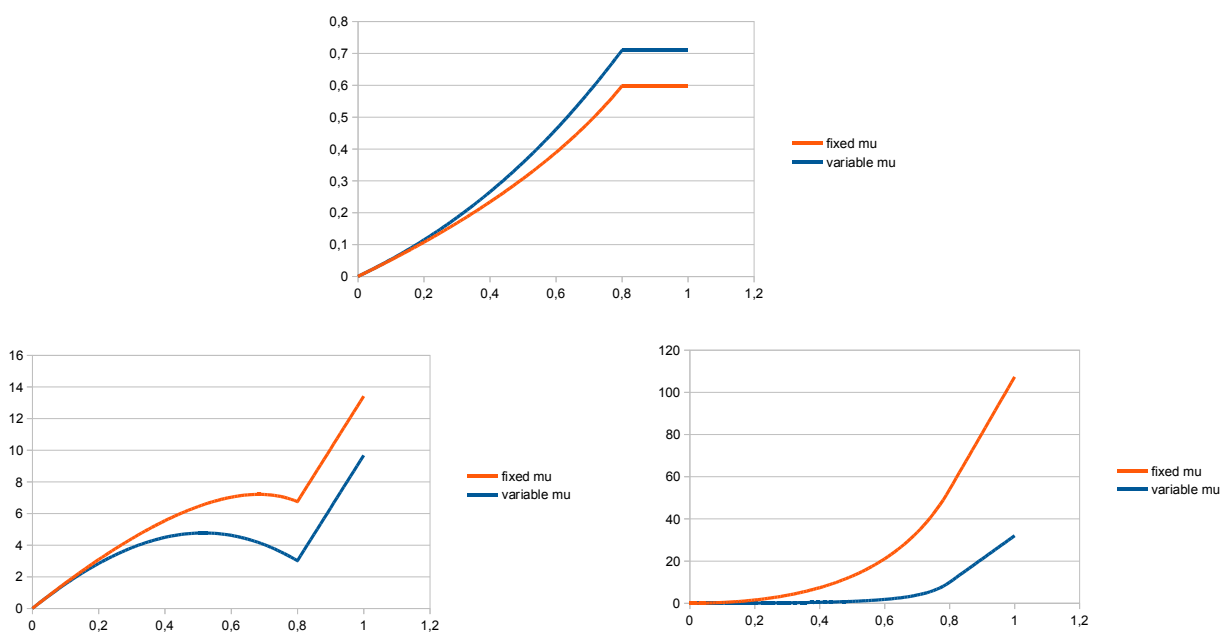

Figure 3: Additive model: Males' strategy $\psi_{\sigma^{T}} / q_{1}$ (top), survivorship $L_{\sigma^{T}} / q_{1}$ (bottom left), and fitness $F_{\mathrm{O}^{\top}} / q_{1}$ (bottom right) as a function of $q / q_{1}$, and for the two variants : limiting P.R.R. (fixed $\mu$ ) or limiting availability of females (variable $\mu$ ) for $\lambda T=8$.

Our models are based on reasonable simplifying assumptions. They allowed us to obtain closed form solutions for all four variants, a useful feature if one wants to discuss the effect of the parameters left: the mean number of males encounter by females in one breeding season, $\lambda T$, and the minimum to maximum survivorship ratio $q_{0} / q_{1}$. The initial operating sex ratio $\lambda / \mu_{0}$ is less significant, only playing as a multiplicative coefficient in males' fitness $F_{\sigma^{7}}$.

As expected, they do yield a large signaling with a correlative large reduction in males' viability, this in the absence of any "decreasing marginal cost of signaling", deemed necessary by [8], but in agreement with Getty [5] [6]. As a matter of fact, in the additive signaling cost model, $\partial^{2} F_{\sigma^{7}} /(\partial q \partial s)=0$, and in the multiplicative signaling cost model, the marginal cost of signaling increases with the male's quality.We claim that the only requirements for what we call the "handicap paradox" to hold are those of our Proposition 1.

Lastly, our models exhibit a very low reproductive efficiency of males of low or average quality. This begs the question of maintenance of quality diversity in the population, a form of the lek paradox [11]. 


\subsubsection{Back to the main hypotheses}

Beyond the hypothesis of the existence of a scalar "quality" for each male, reflecting its survivorship and translating into fitness for the females that mate with him, and of a scalar "signal" associated with quality, the simplicity of the model, and its closed form solution, hinge on 2 main hypotheses:

1. Linear relationship between survivorship and female fitness. Once such a correlation is hypothesized, a linear one is not less realistic than anything else, and obeys in some sort a principle of parsimony, or of simplicity.

2. Uniform distribution of males' quality. Although arbitrary, and difficult or impossible to check in any particular case, it does not seem to be exceedingly unrealistic. Indeed, we need a distribution with compact support, which normal or log-normal distributions cannot provide. This can again be seen as the application of a principle of parsimony.

\subsubsection{Equilibrium selection as an open issue}

The bayesian equilibrium outlined above is not unique. In all cases considered, another bayesian equilibrium pair of strategies is the trivial one where females accept all males, and males do not signal: $\psi_{\uparrow}(t, s)=1$ for all $t, s$, and $\psi_{\sigma^{r}}(q)=0$ for all $q$. Indeed, if females do not expect males to signal, they ignore the signal if any, and choose $\chi(q)=0$. Then males' mating success is not influenced by their signal, which only has a detrimental impact on their fitness, so that they should keep it equal to 0 , and therefore $\psi_{0^{7}}(q)=\chi(q)$.

This raises the issue of whether the nontrivial and trivial Bayesian equilibria attract or repel evolutionary dynamics. This is discussed in the literature [2,3], and is amenable to mathematical analysis in the framework of adaptive dynamics [1] or evolutionary games [17].

\subsubsection{Some qualitative findings}

We have stressed that the females' strategy is essentially the same in all four cases, accepting males with an observed quality no less than $Q(t)$. The literature on sexual selection has often introduced a variable measuring choosiness of females, discussing what cost it imparts on them. In our model with a time dependent threshold strategy as females' optimum strategy, an individual is more choosy than another one if her threshold curve lies "above" the other one's. We obtain a natural choice of threshold in terms of males' quality, since the curve $Q(t)$ does not depend on the female's conjecture $\chi$ nor on the males' behavior. It only depends on the expected 
number $\lambda T$ of encounters with a male in a breeding season and on the minimum to maximum survivorship ratio $q_{0} / q_{1}$.

The larger the expected number $\lambda T$ of encounters per season the choosier the females are, since they have more opportunities, and correlatively, the larger best males' signals are. Correlatively, for a fixed quality, the male fitness $F_{\sigma^{\prime}}$ decreases as the frequency of meetings $\lambda$ increases. The explanation is that when $\lambda$ gets large, females become very choosy, and a male of a given quality is ignored for a longer time during each breeding season; an effect reinforced by the fact that late in the season, the number of available females drops. Perhaps an underappreciated, but rather straightforward, implication of search theory ${ }^{4}$. But of course, this fitness increases proportionally to the inverse initial operational sex ratio $\mu_{0} / \lambda$.

Concerning the ratio $q_{0} / q_{1}$, the larger it is the choosier the females are, since the average male's quality increases with this ratio allowing the females to hope for a better mate. But unexpectedly, in the multiplicative signaling cost model, males' signals decrease when the ratio $q_{0} / q_{1}$ increases.

\subsubsection{An artifact in Grafen's model, and a new model to support his theory}

Grafen [7] similarly noticed that "as $q_{0}$ approaches zero, the general level of advertising increases indefinitely." He quickly proposed an interpretation based on the debatable argument that for a larger $q_{0}$, a male of a given quality has less competitors of lower quality to distinguish himself from. However, there is a simpler and clearer explanation to what actually is an artifact. The point is that both in the multiplicative signaling cost model and in Grafen's, marginal signaling costs vanish as $q$ approaches zero. Indeed, in our multiplicative model, the marginal cost is $-\partial L_{\sigma^{T}} / \partial \sigma=q$, and in Grafen's, where $L_{\sigma^{7}}=q^{\sigma}$ in our notations, it is $-\partial L_{\sigma^{\top}} / \partial \sigma=-q^{\sigma} \log (q)$ (remember that $q \leq q_{1}=1$ in Grafen's). Since in both models, $\lim _{q \rightarrow 0}-\partial L_{\sigma^{\top}} / \partial \sigma=0$ for any $\sigma>0$, a male whose quality is almost zero has little penalty for signaling almost as much as possible, which leads other males to almost full signaling as well. This does not occur in the additive model, since in this case $-\partial L_{\sigma^{T}} / \partial s=1$, which implies that the marginal cost of signaling is never zero, whatever $q$ and $s$. Hence the artifact in the multiplicative model.

In other words, in the multiplicative model akin to Grafen's, we cannot let $q_{0}$ tend to 0 . As a matter of fact, in our formulas, if $q_{0}=0$, then $\varphi_{\mathrm{O}^{7}}(q)=1$ for all $q>0$. Thus, not only is $\varphi_{0^{7}}$ discontinuous at zero, but worse, the survivorship is zero for all males. This is of course an absurd result. Yet, it can be understood in the following way: perturb somewhat the model by assuming that

$$
L_{\sigma^{T}}(q, \sigma)=q(1-\sigma)+\varepsilon
$$

\footnotetext{
${ }^{4}$ We thank an anonymous reviewer for pointing out the relationship with search theory.
} 
for some small positive $\varepsilon$, but keep the constraint $\sigma \leq 1$. And assume $q_{0}=0$. Then a bayesian equilibrium is

$$
\begin{aligned}
& \varphi_{\mathrm{O}^{7}}(0) \text { arbitrary in }[0,1], \\
& \varphi_{\mathrm{O}^{7}}(q)=1 \quad \forall q>0 .
\end{aligned}
$$

As figure 2 shows, this is almost the equilibrium found for very small $q_{0}$. Yet, it was shown above that it depends very specifically on the fact that the multiplicative form hides zero marginal signaling costs when quality tends to zero. Since the additive model has, to our knowledge, no such hidden bias, we believe it makes a better job as a minimal signaling model to start with.

It is nevertheless interesting to give a thought to the multiplicative model game equilibrium for $q_{0}=0$. Should this signal be termed honest [16]? Hardly so in the sense that all males mimic the signal of the best quality males, but yes in the sense that it does not deceive the females. As a matter of fact, in the framework of bayesian equilibrium, "honesty" is merely a consequence of the existence of a unique equilibrium signaling strategy that it is advantageous for the males to use, given the female's conjecture; in essence, existence of a profitable cheating behavior has been defined out by definition 1 and equation (2). In the additive model, the strategy is revealing in the range of qualities below a critical level, and pooling, for high quality males. Yet being unique, it is honest even for these high quality males, revealing that their quality is above the critical level, and it is a sufficient information for the females.

\section{Conclusion}

Any model involves a trade-off between realism on the one hand and mathematical simplicity and tractability on the other hand. We settled somewhat more on the second side of this dilemma than Grafen [8], [7], allowing us to obtain closed form solutions for all four variants investigated. Yet, our models display some interesting features of the bayesian equilibria of signaling games of sexual selection. Of course, we claim no quantitative results, as so many features of all these models are over-simplifications of the real world: not only many phenomena, such as male infighting, have been ignored, but more profoundly, a scalar "quality", moreover taken as a proxy for the benefit a female has to mate with this male, may be an oversimplification of the biological processes at work, and a scalar signal is surely so most often.

At least, comparing our two models let us propose an explanation of the curious fact that previous models forbade a zero minimum quality, and we offer a simple model in which this is not the case. 


\section{A Solving for $\Psi$}

We are interested here in the differential equation (28). For simplicity, we re-write it in terms of the dimensionless $r=q / q_{1}$ and $\psi_{\sigma^{\top}}(q)=q_{1} \psi(r)$. The equation now writes

$$
\psi^{\prime}(r)=\frac{r-\psi(r)}{r(1-r)\left(1-r+\frac{r^{2}}{3}\right)}, \quad \psi(0)=0 .
$$

Write $\psi$ as a power expansion in $r$, i.e.

$$
\psi(r)=\sum_{n=1}^{\infty} \psi_{n} r^{n}, \quad \psi^{\prime}(r)=\sum_{n=1}^{\infty} n \psi_{n} r^{n-1} .
$$

Write

$$
r(1-r)\left(1-r+\frac{r^{2}}{3}\right) \psi^{\prime}(r)=r-\psi(r)
$$

and identify terms of like power in $r$. This yields

$$
\psi_{1}=\frac{1}{2}, \quad \psi_{2}=\frac{1}{3}, \quad \psi_{3}=\frac{1}{6},
$$

and for $n>3$,

$$
3(n+1) \psi_{n}=(n-3) \psi_{n-3}-4 \psi_{n-2}+6(n-1) \psi_{n-1} .
$$

We consider now $\psi(r)+1 / 2$, (i.e. we add a coefficient $\psi_{0}=1 / 2$ ), we compute the 17 first coefficients $\psi_{n}$ and write them in the following table, with six columns labelled by the powers of $r$ as a function of the line number $\ell$ starting at 0 :

\begin{tabular}{c|cccccc}
$\ell$ & $6 \ell-1$ & $6 \ell$ & $6 \ell+1$ & $6 \ell+2$ & $6 \ell+3$ & $6 \ell+4$ \\
\hline 0 & 0 & $\frac{1}{2}$ & $\frac{1}{2}$ & $\frac{1}{3}$ & $\frac{1}{2 \times 3}$ & $\frac{1}{2 \times 3^{2}}$ \\
1 & 0 & $\frac{-1}{2 \times 3^{3}}$ & $\frac{-1}{2 \times 3^{3}}$ & $\frac{-1}{3^{4}}$ & $\frac{-1}{2 \times 3^{4}}$ & $\frac{-1}{2 \times 3^{5}}$ \\
2 & 0 & $\frac{1}{2 \times 3^{6}}$ & $\frac{1}{2 \times 3^{6}}$ & $\frac{1}{3^{7}}$ & $\frac{1}{2 \times 3^{7}}$ & $\frac{1}{2 \times 3^{8}}$
\end{tabular}

This suggests the following general rule:

$$
\psi_{6 \ell-1}=0, \quad 2 \psi_{6 \ell}=2 \psi_{6 \ell+1}=3 \psi_{6 \ell+2}=6 \psi_{\ell+3}=18 \psi_{6 \ell+4}=\frac{(-1)^{\ell}}{3^{3 \ell}}
$$

That these values satisfy the recurrence relation (31) can easily be checked by straightforward, if tedious, calculation. It follows that

$$
\begin{aligned}
\psi(r)+\frac{1}{2} & =\left(\frac{1}{2}+\frac{r}{2}+\frac{r^{2}}{3}+\frac{r^{3}}{6}+\frac{r^{4}}{18}\right) \sum_{\ell=0}^{\infty}\left(\frac{-r^{6}}{27}\right)^{\ell} \\
& =\left(\frac{1}{2}+\frac{r}{2}+\frac{r^{2}}{3}+\frac{r^{3}}{6}+\frac{r^{4}}{18}\right) \frac{1}{1+\frac{r^{6}}{27}}
\end{aligned}
$$


from which formula (29) follows. (As a cross check, we performed a numerical integration with a Runge Kutta scheme of order 4 and a step size of 0.01 , starting from $r=0.05$ and $\psi(0.05)$ estimated with a tenth order power expansion. The result agreed with the closed form formula up to the fifth decimal place at $r=1$.)

\section{References}

[1] Fabio Dercole and Sergio Rinaldi. Analysis of evolutionary processes: the adaptive dynamics approach. Princeton University Publishing, Princeton, N.J., 2008.

[2] Ilan Eshel. Evolutionary and continuous stability. Journal of Mathematical Biology, 108:99-111, 1983.

[3] Ilan Eshel. On the changing concept of evolutionary population stability as a reflection of a changing point of view in the quantitative theory of evolution. Journal of Mathematical Biology, 34:485-510, 1996.

[4] Ronald Aylmer Fisher. The evolution of sexual preferences. Eugenics Review, 7:184-192, 1915.

[5] Thomas Getty. Handicap signalling: when fecundity and viability do not add up. Animal Behaviour, 56:127-130, 1998.

[6] Thomas Getty. Sexually selected signals are not similar to sports handicaps. Trends in Ecology and Evolution, 21:83-88, 2006.

[7] Alan Grafen. Biological signals as handicaps. Journal of Theoretical Biology, 144:517-546, 1990.

[8] Alan Grafen. Sexual selection unhandicapped by the fisher process. Journal of Theoretical Biology, 144:473-516, 1990.

[9] Peter L. Hurd and Magnus Enquist. A strategic taxonomy of biological communication. Animal Behaviour, 70:1155-1170, 2005.

[10] Rufus A. Johnstone and Alan Grafen. Dishonesty and the handicap principle. Animal Behaviour, 46:759-764, 1993.

[11] Mark Kirkpatrick and Michael J. Ryan. The evolution of mating preferences and the paradox of the lek. Nature, 350:33-38, 1991. 
[12] Hanna Kokko, Michael D. Jennions, and Robert Brooks. Unifying and testing models of sexual selection. Annual Review of Ecology, Evolution, and Systematics, 37:43-66, 2006.

[13] Russell Lande. Models of speciation by sexual selection on polygenic traits. Proceedings of the National Academy of Science of the USA, 78:3721-3725, 1981.

[14] N. Nur and O. Hasson. Phenotypic plasticity and the handicap principle. Journal of Theoretical Biology, 110:257-297, 1984.

[15] Stephen R. Proulx, Troy Day, and Locke Rowe. Older males signal more reliably. Poceedings of the Royal Society of London, 269:2291-2299, 2002.

[16] Szalbolcs Számadó. The cost of honesty and the fallacy of the handicap principle. Animal Behaviour, 81:3-10, 2011.

[17] Thomas L. Vincent and Joel S. Brown. Evolutionary Game Theory, Natural Selection and Darwinian Dynamics. Cambridge University Press, Cambridge, U.K., 2006.

[18] Amots Zahavi. Mate selection - a selection for the handicap. Journal of Theoretical Biology, 53:205-214, 1975. 